\title{
Dandruff Associated Changes in Epidermal Morphology
}

Pople JE, Bhogal RK, Moore AE \& Jenkins G Unilever R\&D Colworth, Colworth Science Park, Bedford, UK.

\section{Introduction}

Dandruff is a very common scalp condition reportedly affecting upwards of $50 \%$ of the global adult population (1). Host factors such as sebum production seem to play a key role as dandruff is only present in the post-pubertal

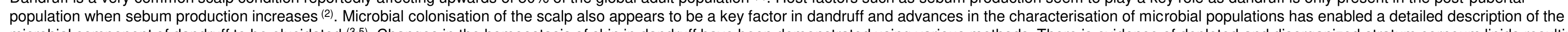

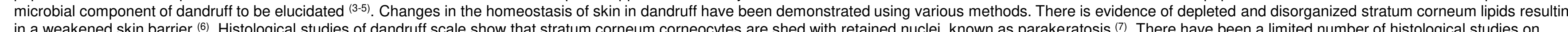
in a weakened skin barrier ${ }^{2}$. Histological studies of dandruff scale show that stratum corneum corneocytes are shed with retained nuclei, known as parakeratosis $(7)$. There have been a limited number of histological studies on

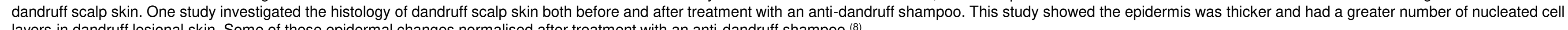
layers in dandruff lesional skin. Some of these epidermal changes normalised after treatment with an anti-dandruff shampoo (8).

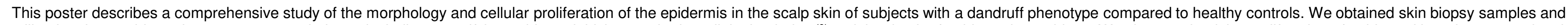

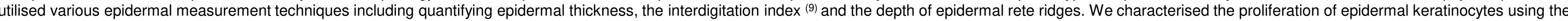
well-established cellular proliferation biomarker Ki67 (10).

\section{Study Design}

An ethically approved clinical study to investigate the characteristics of scalp skin in dandruff was conducted. Female subjects (mean age 39.8 + - 15.4) were recruited and their scalp health graded using total weighted head score (11) and were classified as either healthy (score $\leq 8$ ) or dandruff sufferers (score $\geq 32$ ). $4 \mathrm{~mm}$ scalp skin punch biopsies were collected from 22 healthy subjects and 21 dandruff sufferers from both lesional and non-lesional site.

\section{Methods}

Biopsies were snap frozen and $10 \mu \mathrm{m}$ sections cut, stained using haematoxylin and eosin and images across the whole epidermis gathered using bright field microscopy. Epidermal thickness was calculated using an in house algorithm. The interdigitation index was calculated followed the method described by Timár et al (9). Epidermal rete ridge depth was calculated for all visible ridges using ImageJ. Immunohistochemistry for the proliferation marker Ki67 was completed. Image analysis of the expression was performed using Halo® image analysis system and CytoNuclear quantification statistically using JMP 11 (SAS, UK)

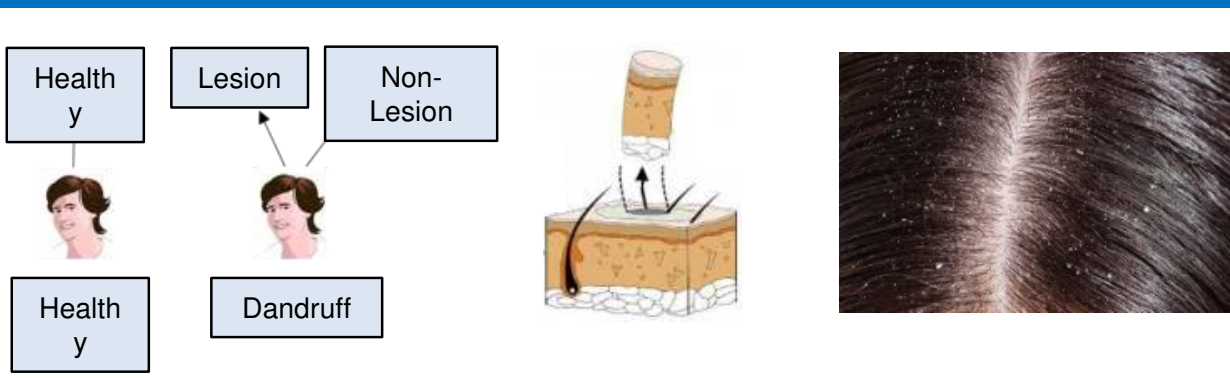

Dandruff skin shows significant changes in epidermal morphology
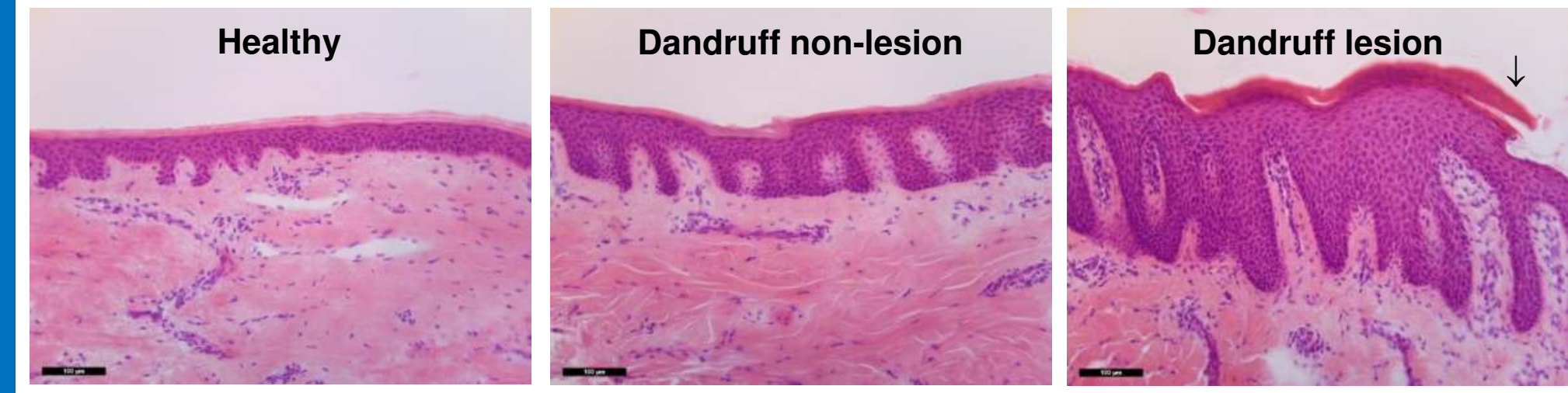

The epidermis was significantly thicker in dandruff lesional skin, mean $95.08 \mu \mathrm{m}$, compared to healthy scalp skin, mean $57.08 \mu \mathrm{m}$

The dermal epidermal junction was significantly more convoluted in dandruff lesional skin compared to healthy scalp as measured by the interdigitation index.

Epidermal rete ridges were significantly deeper in dandruff skin, mean $83.39 \mu \mathrm{m}$ compared to healthy scalp skin, mean $47.70 \mu \mathrm{m}$

Non-lesional dandruff skin also showed changes in epidermal morphology suggesting it isn't completely healthy

Dandruff lesional skin corneocyte flakes containing parakeratotic nuclei $(\downarrow)$
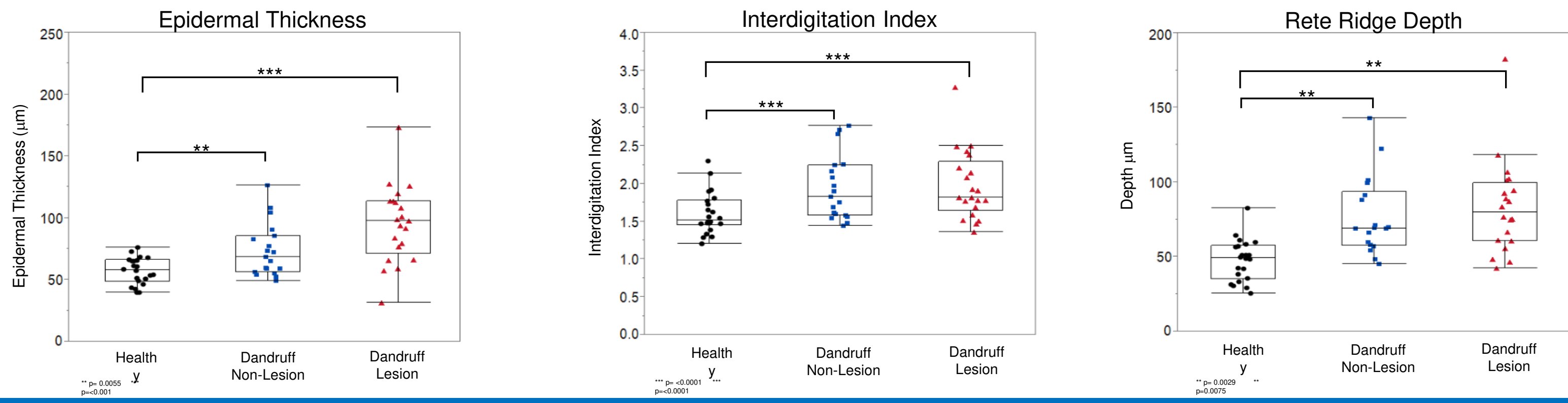

Dandruff skin shows increased expression of the proliferation marker Ki67
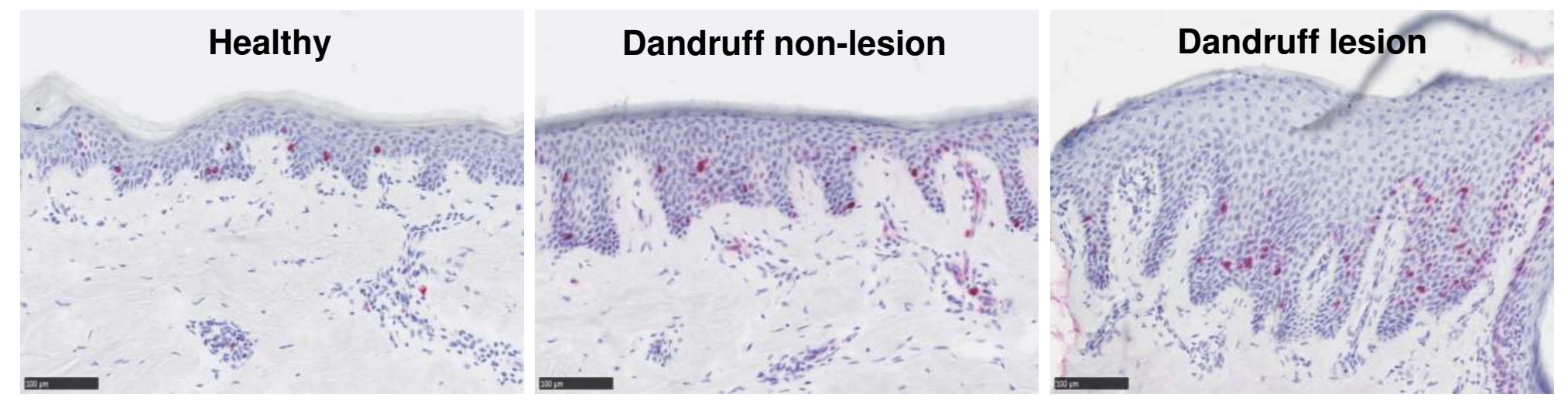

Dandruff lesional skin contained a significantly higher percentage of proliferating Ki67 positive keratinocytes, mean $11.84 \%$, compared to healthy controls, mean $4.22 \%$ Dandruff lesional skin had a higher expression level of Ki67 protein, mean 27.86, compared to healthy scalp skin, mean 9.05 as measured using $\mathrm{H}$ score

This confirms that in dandruff epidermal keratinocytes are in a hyper-proliferative state

\section{Conclusions}

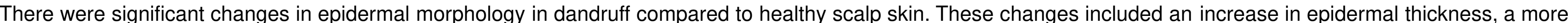

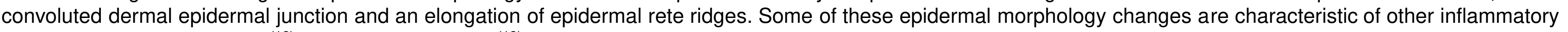
skin conditions like psoriasis ${ }^{(12)}$ and atopic dermatitis ${ }^{(13)}$. Changes were also evident in dandruff non-lesional skin suggesting it wasn't healthy. 